\title{
FILLING RANDOM CYCLES
}

\author{
FEDOR MANIN
}

\begin{abstract}
We compute the asymptotic behavior of the average-case filling volume for certain models of random Lipschitz cycles in the unit cube and sphere. For example, we estimate the minimal area of a Seifert surface for a model of random knots first studied by Millett. This is a generalization of the classical Ajtai-Komlós-Tusnády optimal matching theorem from combinatorial probability. The author hopes for applications to the topology of random links, random maps between spheres, and other models of random geometric objects.
\end{abstract}

\section{INTRODUCTION}

1.1. Main results. This paper introduces a new kind of average-case isoperimetric inequality. Given a $k$-cycle $Z$ on $\left([0,1]^{n}, \partial[0,1]^{n}\right)$, in any of a number of geometric measure theory senses, its filling volume $F V(Z)$ is the minimal mass of a chain whose boundary is $Z$. The well-known Federer-Fleming isoperimetric inequality [FF] states that for all $k$-cycles $Z$,

$$
F V(Z) \leq C_{n, k} \operatorname{mass}(Z)^{\frac{k+1}{k}} \quad \text { and } \quad F V(Z) \leq C_{n, k} \operatorname{mass}(Z) ! 1
$$

However, one might expect that most cycles of given mass are much easier to fill.

Unfortunately, as explained to the author by Robert Young, a geometrically meaningful probability measure on the space of all cycles of mass $\leq N$ may be too much to ask for. The issue is one of picking a scale: say we are trying to build a random 1-Lipschitz curve in a finite-dimensional space. If the curve is to fluctuate randomly at scale $\varepsilon$, then over time 1 it will only travel a distance on the order of $\sqrt{\varepsilon}$. Thus there is no way of ensuring random behavior in a scale-free way. This idea of decomposing a finite-mass cycle into pieces at different scales can be made precise using the notion of a corona decomposition, as in [Jones] (in dimension 1) and Young] (in higher dimensions).

On the other hand, there are a number of ways of, and a number of motivations for, building random "space-filling" cycles of mass $O(N)$ which look essentially trivial on balls of radius $N^{-1 / n}$. Our main theorems characterize three models of this form which exhibit similar isoperimetric behavior, and we hypothesize that this behavior should be generic for models which are random at many scales, including the largest - an idea which may have a precise Fourier-analytic formulation.

This isoperimetric behavior is described in codimension $d$ by the function

$$
\operatorname{AKT}_{d}(N)= \begin{cases}\sqrt{N} & \text { if } d=1 \\ \sqrt{N \log N} & \text { if } d=2 \\ N^{(d-1) / d} & \text { if } d \geq 3\end{cases}
$$

\footnotetext{
${ }^{1}$ The first inequality dominates when $\operatorname{mass}(Z)<<1$, the second when $\operatorname{mass}(Z)>>1$.
} 
Theorem A. Let $Z$ be a $k$-cycle on $S^{n}$ obtained by sampling $N$ oriented great $k$ spheres independently from the uniform distribution on the oriented Grassmannian $\widetilde{G r}_{k+1}\left(\mathbb{R}^{n+1}\right)$. Then there are constants $C>c>0$ depending on $n$ and $k$ such that

$$
c \operatorname{AKT}_{n-k}(N)<\mathbb{E}(F V(Z))<C \operatorname{AKT}_{n-k}(N) .
$$

Moreover, $F V(Z)$ is concentrated around its mean: there are constants $C_{1}, C_{2}>0$ depending on $n$ and $k$ such that

$$
\text { for every } r>0, \mathbb{P}[|F V(Z)-\mathbb{E}(F V(Z))| \geq r] \leq C_{1} \exp \left(-C_{2} \sqrt{N} r\right)
$$

From (1.2) we see that the spread of the distribution around the mean is at most on the order of $\sqrt{N}$; in codimensions $d=n-k \geq 2$, this is small compared to the mean:

$$
\text { for every } \varepsilon>0, \frac{|F V(Z)-\mathbb{E}(F V(Z))|}{\mathbb{E}(F V(Z))} \leq \varepsilon \text { with high probability as } N \rightarrow \infty \text {. }
$$

If a model of random codimension- $d$ cycles of mass $O(N)$ satisfies (1.1) and (1.2), we say it exhibits AKT statistics, in honor of Ajtai, Komlós, and Tusnády, who discovered this phenomenon in the case of zero-cycles.

By rescaling the picture, we can make this result more interpretable. Let $R=$ $N^{1 /(n-k)}$. Then the corresponding process in the $n$-sphere of radius $R$ generates a cycle of mass $\Theta\left(R^{n}\right)$ which is evenly spread throughout the sphere, so that a 1-ball intersects one of the great $k$-spheres in $Z$ on average. With that rescaling, the mass of an optimal filling becomes

$$
\begin{cases}\Theta\left(R^{n} \sqrt{R}\right) & \text { if } k=n-1 \\ \Theta\left(R^{n} \sqrt{\log R}\right) & \text { if } k=n-2 \\ \Theta\left(R^{n}\right) & \text { if } k \leq n-3\end{cases}
$$

Informally speaking, to meet its match, the average point in $Z$ has to travel a distance $\Theta(\sqrt{R})$ (in codimension 1), $\Theta(\sqrt{\log R})$ (in codimension 2), or $\Theta(1)$ (otherwise) times the distance to its closest neighbor.

We also prove similar results for the cube:

Theorem B. Let $Z$ be a relative $k$-cycle on $\left([0,1]^{n}, \partial[0,1]^{n}\right)$ obtained by sampling $N$ planes independently from the uniform distribution on the space $Y$ of oriented $k$-planes which intersect $[0,1]^{n}$ nontrivially. Then $Z$ exhibits AKT statistics.

There may be reasonable disagreement as to which distribution is the uniform one in this context; to prove (1.1) it suffices to require that it be uniform on each subset of $Y$ (isometric to two copies of a polytope) consisting of parallel planes, but to prove (1.2) we also need to assume that it behaves reasonably with respect to the manifold structure on $Y$ (for example, is a positive density or has finite support).

In fact, the only thing used here about $Z$ is that almost all of its "slices" along coordinate $k$-planes consist of $O(N)$ independent uniformly distributed points. This means that there are a number of other possible models that can be fit into this framework. However, the following requires a separate proof:

Theorem C. Let $\left\{M_{N}\right\}$ be a sequence of $k$-dimensional oriented pseudomanifolds with $N$ vertices and at most $L$ simplices incident to any given simplex. Let $Z$ be a $k$-cycle on $[0,1]^{n}$ obtained by sending each vertex of $M_{N}$ to a uniformly random point in $[0,1]^{n}$ and extending linearly. Then $Z$ exhibits AKT statistics. 
In the context of this theorem, the constants in (1.1) depend on $n, k$, and $L$, but not on the shapes of the pseudomanifolds (which can therefore also be randomized). The case $k=1, n=3$ describes the "random jump" model of random knots and links introduced by Millett [Mil]. Moreover, by a theorem of Hardt and Simon [HS], the optimal filling of such a knot or link (after a slight rounding of corners) is a $C^{1}$ embedded surface. In particular:

Corollary 1.3. For some $C>c>0$, the minimal Seifert surface of a knot produced using $N$ random jumps has area between $c \sqrt{N \log N}$ and $C \sqrt{N \log N}$ with high probability.

1.2. Motivation. The methods we use to prove Theorems $\mathrm{A}, \mathrm{B}$, and $\mathrm{C}$ can be easily extended to other i.i.d. samples of simple shapes on various spaces. However, the investigation is mainly motivated by the desire to analyze topological invariants of random geometric objects such as links and maps. Models of such objects tend to produce random cycles which are similarly trivial at small scales, but are more difficult to sample because they cannot be easily written in terms of i.i.d. parameters.

Random knots and links. There have been a number of proposed models of random knots and links; see [E-Z] for a detailed survey. Several of these models are "spatial" in the sense that they produce random knotted curves in space, and one supposes that these may exhibit AKT statistics for filling area. As mentioned above, we show this for Millett's random jump model, but it may also be true for random polygonal walks with shorter segments as well as random grid walks, perhaps with some restrictions on segment length.

Given two random curves in a certain model, one may want to understand the distribution of their linking number. Since this will usually be zero on average, the first interesting question is about the second moment. Linking number can be computed as the intersection number of one curve with a filling of the other, thus one may expect that two random curves of length $N$ which exhibit AKT statistics have expected squared linking number $\sim N \log N$ or $\sim N \sqrt{\log N}$.

However, this is not the case for the Millett model: the second moment of the linking number between two random jump curves of length $N$ is $\sim N\left[\mathrm{ABD}^{+}, \mathrm{FK}\right.$. Similarly, one may take the setup of Theorem $\mathrm{A}$ for $k=1$ and $n=3$ as a model of a random link and try to understand the total linking number, that is, the sum of the signed linking numbers of all pairs of circles. This is then the intersection number of the chain with its own filling. Here it is easy to see (as pointed out by Matthew Kahle) that the second moment of the distribution is once again $\sim N$.

In both cases, this seeming incongruity perhaps boils down once again to the issue of multiple scales: random jump curves and great circles only "see" the largest scales, but the lower bound on filling volume in codimension 2 comes from looking on many different scales at once. One may perhaps get a different answer most easily by analyzing the linking number of an asymmetric model: a random jump curve and a random walk of total length $N$ made of smaller segments.

In Tanaka, Marko, the second moment is computed for the linking number of two random walks; normalizing so that these walks have length $N$ and expected diameter 1 , this second moment again becomes $\sim N$. In this model, however, randomness happens at scale $\sim 1 / N$, so it is not expected to exhibit AKT statistics. 
Random maps. Another way of producing a random (framed) link is as the preimage of a generic point under a random map $f: S^{3} \rightarrow S^{2}$. In fact, the self-linking number of this link is the Hopf invariant of the map, which is itself a natural subject for investigation since it is a complete topological invariant of such maps.

One natural model of $L$-Lipschitz random maps is a uniformly random simplicial map from a triangulation of $S^{3}$ at scale $\sim L$ to a tetrahedron. The maximal selflinking number of such a map is $\Theta\left(L^{4}\right)$, cf. Gro]; on the other hand, the heuristics above would suggest that the second moment of the linking number of the random model is between $L^{3}$ and $L^{3} \log L$.

These ideas may have applications in topological and geometric data analysis, see [FKW].

1.3. Methods. The $k=0$ cases of Theorems $\mathrm{A}$ and $\mathrm{B}$ are, up to minor adjustments, a classical theorem in combinatorial probability:

Theorem 1.4 (Ajtai, Komlós, and Tusnády AKT). Let $\left\{X_{1}, \ldots, X_{N}\right\}$ and $\left\{Y_{1}, \ldots, Y_{N}\right\}$ be two sets of independent, uniformly distributed random points in $[0,1]^{d}$, and let $L$ be the transportation cost between $\left\{X_{i}\right\}$ and $\left\{Y_{i}\right\}$, that is, the total length of an optimal matching. Then there are constants $0<c_{d}<C_{d}$ such that with high probability,

$$
c_{d} \operatorname{AKT}_{d}(N)<L<C_{d} \operatorname{AKT}_{d}(N) .
$$

Since the original geometric proof in AKT of the most subtle case $d=2$, this and related results have been proved many other times, often by applying Fourier analysis; see [BL2] for further references and [Tal3] for a detailed treatment of certain analytic approaches. Another beautiful geometric proof of the upper bound on the sphere is due to Holden, Peres, and Zhai [HPZ].

The proofs of Theorems $\mathrm{A}$ and $\mathrm{B}$ in general are obtained by applying the $k=0$ results to $(n-k)$-dimensional slices of the cube and sphere. This is the reason that the results depend only on the codimension, and for the critical nature of codimension 2. The lower bound in (1.1) is obtained directly by integrating the lower bounds on these slices. The upper bound is obtained via a dual result on differential forms; this kind of technique was already used in [AKT for the proof of the lower bound for the square. Finally, (1.2) is proved using the notion of concentration of measure due originally to Gromov and Milman [GM]; see [Led] for an extensive modern treatment.

Theorem $\mathrm{C}$ is proved similarly, except that slices no longer consist of i.i.d. points. Even this small amount of dependence complicates the argument considerably. We use ad hoc combinatorial arguments to overcome this, but one might hope to generalize, for example by applying a variant of Stein's method, to a version of Theorem 1.4 in the presence of dependence (one approach, which only gives upper bounds, is discussed in [BL2, §5]).

Structure of the paper. Section 2 introduces necessary ideas and results from geometric measure theory, and Section 3 discusses the classical AKT theorem. In Sections 4 and 5, the upper and lower bounds in Theorems $\mathrm{A}$ and B are proved using tools that may generalize to other models of random cycles. In Section 6, we discuss the extra ideas needed to prove Theorem C. Finally, Section 7 discusses the concentration of the distributions in these theorems around their mean. 
Acknowledgements. I would like to thank Matthew Kahle and Robert Young for a large number of helpful discussions over a span of three years. Yevgeny Liokumovich provided a crucial reference; Shmuel Weinberger asked a question which inspired Theorem [C and gave other helpful comments. Finally, I would like to thank both Robert Young and the anonymous referee for calling out a great deal of sloppy and lazy writing in the initial draft. I was partially supported by NSF individual grant DMS-2001042.

\section{Definitions AND PRELIMINARIES}

2.1. Cycles and currents. There are a number of useful ways to define chains and cycles from the point of view of topology and geometric measure theory. Algebraic topology typically uses singular $k$-chains: formal linear combinations of continuous maps from the $k$-simplex to a topological space $X$ ("singular simplices"). We will usually restrict our attention to Lipschitz simplices (that is, requiring the maps to be Lipschitz) on a Riemannian manifold $M$. By Rademacher's theorem, a Lipschitz simplex $\sigma: \Delta^{k} \rightarrow M$ is differentiable almost everywhere and so has a well-defined volume or mass,

$$
\operatorname{mass}(\sigma)=\int_{\Delta^{k}} \sigma^{*} d \operatorname{vol}_{M}
$$

We can then extend by linearity to define the mass of a Lipschitz chain.

A more general notion of chain is that of a normal current. A $k$-dimensional current on a manifold $M$ is simply a functional on (smooth) differential forms, which we think of as integration over the current. For example:

- Every Lipschitz chain $T$ defines a current via $\omega \mapsto \int_{T} \omega$.

- Every compactly supported $(n-k)$-form $\alpha \in \Omega^{n-k}(M)$ defines a current via $\omega \mapsto \int_{M} \alpha \wedge \omega$.

We will write the value of $T$ on $\omega$ either as $T(\omega)$ or as $\int_{T} \omega$, since currents should be thought of as generalized domains of integration. The boundary operator is defined via Stokes' theorem: for a current $T$,

$$
\partial T(\omega)=T(d \omega) .
$$

The mass of a $k$-current $T$ on $M$, which agrees with the same notion on Lipschitz chains, is defined to be

$$
\operatorname{mass}(T)=\inf \left\{T(\omega): \omega \in \Omega^{k}(M) \text { and }\|\omega\|_{\infty}=1\right\} .
$$

Here $\|\omega\|_{\infty}$ is the supremum of the value of $\omega$ over all frames of unit vectors. For a general current, the mass of course need not be finite. A current $T$ is normal if $T$ and $\partial T$ both have finite mass; in particular any cycle (current with empty boundary) of finite mass is normal.

2.2. Fillings and duality. Now, if $S$ is a normal current such that $\partial S=T$, we call it a filling of $T$. The filling volume of $T$ is

$$
F V(T)=\inf \{\operatorname{mass}(S) \mid \partial S=T\},
$$

which is always finite by the work of Federer and Fleming. The following is an instance of the Hahn-Banach theorem: 
Proposition 2.1. Let $M$ be a manifold. Then a normal $k$-current $T$ in $M$ with $\partial T=0$ has a filling of mass $c$ if and only if for every $\omega \in \Omega^{k}(M)$ with $\|d \omega\|_{\infty} \leq 1$, $\int_{T} \omega \leq c$.

More generally, for any closed set $A \subset M$, write $\Omega^{k}(M, A)$ for the vector space of forms whose restriction to $A$ is zero. Let $T$ be a normal $k$-current with $\partial T$ supported on $A$, that is, such that $\int_{\partial T} \alpha=0$ for any $(k-1)$-form $\alpha \in \Omega^{k-1}(M, A)$. Then $T$ has a filling relative to $A$ (that is, a $(k+1)$-current $S$ such that $\partial S-T$ is supported on $A)$ of mass $c$ if and only if for every $\omega \in \Omega^{k}(M, A)$ with $\|d \omega\|_{\infty} \leq 1, \int_{T} \omega \leq c$.

In other words, the filling volume of a cycle $T$ can be redefined as

$$
F V(T)=\sup \left\{\int_{T} \omega \mid \omega \in \Omega^{k}(M) \text { such that }\|d \omega\|_{\infty} \leq 1\right\}
$$

in both the absolute and the relative case. Our proofs of the upper bounds in Theorems $\mathrm{A}$ and $\mathrm{B}$ will be based on this proposition rather than constructing fillings directly.

Of course, knowing that a nice Lipschitz cycle has a filling which is a normal current is not very satisfying - after all, normal currents can still be very strange. Luckily, given a normal current filling, we can upgrade it to a Lipschitz chain (at the cost of multiplying the mass by a constant) using the following classical theorem:

Theorem 2.2 (Federer-Fleming deformation theorem [FF, Thm. 5.5]). There is a constant $\rho(k, n)=2 n^{2 k+2}$ such that the following holds. Let $T$ be a normal current in $\mathbf{N}_{k}\left(\mathbb{R}^{n}\right)$. Then for every $\varepsilon>0$ we can write $T=P+Q+\partial S$, where

(1) $\operatorname{mass}(P) \leq \rho(k, n) \operatorname{mass}(T)$.

(2) $\operatorname{mass}(Q) \leq \varepsilon \rho(k, n) \operatorname{mass}(\partial T)$

(3) $\operatorname{mass}(S) \leq \varepsilon \rho(k, n) \operatorname{mass}(T)$.

(4) $P$ is a polyhedral cycle which can be expressed as an $\mathbb{R}$-linear combination of $k$-cells in the cubical unit lattice in $\mathbb{R}^{n}$.

(5) If $T$ is a Lipschitz chain, then so are $Q$ and $S$.

(6) If $\partial T$ is a Lipschitz chain, then so is $Q$.

If $T$ is a normal current filling a Lipschitz chain $\partial T$, then $P+Q$ is a Lipschitz chain filling $T$ whose mass is only greater by a multiplicative constant $\rho(k, n)$.

It is not hard to upgrade the deformation theorem to manifolds, although the resulting constants will depend on the manifold and its metric; see for example $\mathrm{EPC}^{+}$, Theorem 10.3.3].

2.3. Slicing. An important property of normal currents, introduced in [FF, §3], is the ability to take "slices" by hyperplanes to produce currents in lower dimensions. We follow the exposition of F. Morgan [Mor, 4.11], who follows Federer [Fed, §4.2.1].

Let $u: M \rightarrow \mathbb{R}$ be a Lipschitz function on a manifold $M$. Given a $k$-current $T$ on $M$ and a differential $r$-form $\omega$, define the $(k-r)$-current $T\llcorner\omega$ by

$$
T\llcorner\omega(\eta)=T(\omega \wedge \eta) .
$$

In particular, this makes sense when $\omega$ is a measurable function, for example the characteristic function $\chi_{A}$ of a set $A$. In that case we can write $T\left\llcorner A=T\left\llcorner\chi_{A}\right.\right.$ for the restriction of $T$ to $A$.

Given a Lipschitz function $u: M \rightarrow \mathbb{R}$, the slice of $T$ at $u(x)=r$ is defined by

$$
T \cap\{u(x)=r\}=(\partial T)\llcorner\{u(x)>r\}-\partial(T\llcorner\{u(x)>r\}) .
$$


If $T$ is a normal current, then $T \cap\{u(x)=r\}$ is a normal current for almost all $r$. In addition, we have the following standard properties:

(1) If $T$ is defined by integration over a (rectifiable) set $X \subset M$, then $T \cap\{u(x)=$ $r\}$ is defined by integration over $M \cap\{u(x)=r\}$.

(2) $\partial T \cap\{u(x)=r\}=-\partial(T \cap\{u(x)=r\})$.

(3) $\operatorname{mass}(T) \geq \frac{1}{\operatorname{Lip} u} \int_{-\infty}^{\infty} \operatorname{mass}(T \cap\{u(x)=r\}) d r$.

A quick calculation from the definitions yields an additional property:

Proposition 2.4. If $\omega$ is a $(k-1)$-form, then

$$
T(d u \wedge \omega)=-\int_{-\infty}^{\infty}(T \cap\{u(x)=r\})(\omega) d r .
$$

Proof. Start with the equalities

$$
\begin{aligned}
& \partial\left(T\llcorner u)(\omega)=\int_{-\infty}^{\infty} \partial(T\llcorner\{u(x)>r\})(\omega) d r\right. \\
& \left(\partial T\llcorner u)(\omega)=\int_{-\infty}^{\infty}((\partial T)\llcorner\{u(x)>r\})(\omega) d r .\right.
\end{aligned}
$$

Subtract one from the other; then applying Stokes' theorem and the Leibniz rule on the left and (2.3) on the right, we get the desired identity.

In particular, by inductively slicing in different directions, we get the following:

Proposition 2.5. Let $1 \leq k \leq m \leq n$, and let $T$ be an $m$-dimensional current on $[0,1]^{n}$. Given $\vec{x}=\left(x_{1}, \ldots, x_{k}\right) \in \mathbb{R}^{k}$, let $P_{\vec{x}}$ be the plane $\{\vec{x}\} \times \mathbb{R}^{n-k}$. Then there are $(m-k)$-dimensional currents $T \cap P_{\vec{x}}$, normal for almost all $\vec{x}$, such that

$$
\begin{gathered}
\partial\left(T \cap P_{\vec{x}}\right)=\partial T \cap P_{\vec{x}} \\
\operatorname{mass}(T) \geq \int_{[0,1]^{k}} \operatorname{mass}\left(T \cap P_{\vec{x}}\right) d \vec{x} .
\end{gathered}
$$

In addition, given an $m$-index $I \subset\{1, \ldots, n\}$ and a function $f:[0,1]^{n} \rightarrow \mathbb{R}$,

$$
\int_{T} f d x_{I}=(-1)^{m} \int_{[0,1]^{c}}\left(\int_{T \cap P_{\vec{x}}} f\right) d \vec{x} .
$$

\section{A variation on the Ajtai-Komlós-TusnÁdy theorem}

The results of this paper are a generalization of Theorem 1.4. Properly, the theorem of Ajtai, Komlós, and Tusnády [AKT] is in the case $n=2$; their paper also asserts the case $n \geq 3$, which is easy and later proved and extended in several directions by Talagrand [Tal1, Tal2]. The $n=1$ case is elementary, and the proof along with a vast array of strengthenings and generalizations can be found in BL1] by Bobkov and Ledoux. Here we need a slight variation.

Theorem 3.1. Generate a cycle $Z$ of mass $N$ in $C_{0}\left([0,1]^{n}, \partial[0,1]^{n}\right)$ by selecting $N$ independent, uniformly distributed points in $[0,1]^{n} \times\{+1,-1\}$. Then there are constants $0<c_{n}<C_{n}$ such that

$$
c_{n} \operatorname{AKT}_{n}(N) \leq \mathbb{E}(F V(Z)) \leq C_{n} \operatorname{AKT}_{n}(N) .
$$


Remark 3.2. Suppose that $D$ is a Riemannian ball diffeomorphic to $[0,1]^{n}$ and has a volume form. Then by the main theorem of BMPR] (extending results of Moser [Moser] and Banyaga [Bany]), there is a diffeomorphism between the two which multiplies the volume form by a constant. Therefore Theorem 3.1 also holds with respect to Lebesgue measure on $D$, with constants $0<c_{D}<C_{D}$ depending on the ratio of the volumes and the bilipschitz constant of this diffeomorphism.

Moreover, given a smooth family of Riemannian balls, BMPR indicates that there is a smooth family of such diffeomorphisms. Therefore, if the family is compact, one can find uniform constants for the whole family.

Proof. There are two differences here from the results as they are typically presented in the probability literature, where the problem consists of matching two sets of random points of the same cardinality: first, the number of positive and negative points may not match; second, we are allowed to match points to the boundary as well as to points of the opposite orientation 2 We briefly explain how to modify the original proofs to deal with this.

Clearly, the possibility of matching to the boundary cannot make the upper bounds worse. Let's say without loss of generality there are more positive points. To obtain the upper bound for $n \geq 2$, we may simply ignore some arbitrary set of "extra" positive points, matching all the others first. By the central limit theorem, the expected number of extra points is $O(\sqrt{N})$, so the extra mass generated by matching them all to the boundary of the cube does not change the asymptotic answer.

For the lower bound in the case $n=2$, we use the same stratagem of ignoring the "extra" points to create a new cycle $Z^{\prime}$ with an equal number of positive and negative points. From the original proof in $\mathrm{AKT}$, we know that there is a 1-Lipschitz function $f:[0,1]^{2} \rightarrow \mathbb{R}$ which is zero on $\partial[0,1]^{2}$ and such that $\int_{Z^{\prime}} f \geq c \sqrt{N \log N}$ with high probability. Since with high probability the number of extra points is $<<\sqrt{N \log N}$, and the values of $f$ lie between $-1 / 2$ and $1 / 2$, we also know that $\int_{Z} f \geq c \sqrt{N \log N}$ with high probability.

The lower bound in the case $n \geq 3$ is easy to see: conditional on any distribution of the positive points, most negative points will be at distance $\geq c N^{-1 / n}$ from every positive point and the boundary, where $c>0$ is a constant depending on $n$.

In the case $n=1$, the filling is unique up to a constant: the unique filling $F$ supported away from zero has density $\int_{0}^{x} Z$ at $x \in[0,1]$. We use arguments found in $[\mathrm{BL1}, \S 3]$ to give estimates on $\mathbb{E}(\operatorname{mass} F)$.

The upper bound is a simple calculation:

$$
\begin{aligned}
\mathbb{E}(\operatorname{mass} F) & =\int_{0}^{1} \mathbb{E}\left(\left|\int_{0}^{x} Z\right|\right) d x \\
& \leq \int_{0}^{1} \sqrt{\operatorname{Var}\left(\int_{0}^{x} Z\right)} d x=\frac{\sqrt{N}}{2} .
\end{aligned}
$$

The lower bound comes from the following classical fact, found in [BL1] as Lemma 3.4:

\footnotetext{
${ }^{2}$ In fact, a somewhat similar, but more complicated modification was studied by Shor $\underline{\text { Shor. }}$.
} 
Lemma 3.3. Given independent mean zero random variables $\xi_{1}, \ldots, \xi_{N}$,

$$
\mathbb{E}\left(\left|\sum_{k=1}^{N} \xi_{k}\right|\right) \geq \frac{1}{2 \sqrt{2}} \mathbb{E}\left(\left(\sum_{k=1}^{N} \xi_{k}^{2}\right)^{1 / 2}\right) .
$$

Let $\left(X_{k}, \sigma_{k}\right) \in[0,1] \times\{+1,-1\}$ be the $k$ th chosen point. Then applying the lemma to $\xi_{k}=\sigma_{k} \chi_{\left\{X_{k} \leq x\right\}}$, we get

$$
\begin{aligned}
\mathbb{E}\left(\left|\int_{0}^{x} Z\right|\right) & \geq \frac{1}{2 \sqrt{2}} \mathbb{E}\left(\left(\sum_{k=1}^{N} \xi_{k}^{2}\right)^{1 / 2}\right) \\
& \geq \frac{1}{2 \sqrt{2}}\left(\sum_{k=1}^{N}\left(\mathbb{E}\left(\left|\xi_{k}\right|\right)\right)^{2}\right)^{1 / 2}=\frac{1}{2 \sqrt{2}} \sqrt{N} x,
\end{aligned}
$$

and therefore $\mathbb{E}(\operatorname{mass} F) \geq \sqrt{N / 32}$.

\section{PROOF OF THE UPPER BOUND}

To prove the upper bound in Theorems $\mathrm{A}$ and $\mathrm{B}$, we will use Stokes' theorem; that is, we use the fact that for a cycle $Z \in C_{k}(M, A)$,

$$
F V(Z)=\sup \left\{\int_{Z} \alpha: \alpha \in \Omega^{k}(M, A) \text { such that }\|d \alpha\|_{\infty}=1\right\} .
$$

In fact, since $Z$ is a cycle, $\int_{Z} \alpha$ only depends on $\omega=d \alpha$. To bound this quantity, we first note that any $\omega \in \Omega^{k+1}\left([0,1]^{n}\right)$ can be decomposed into a sum of "basic" forms of the form

$$
\omega_{I}(x) d x_{i_{1}} \wedge \cdots \wedge d x_{i_{k+1}},
$$

where $\omega_{I}$ is a function $\mathbb{R}^{n} \rightarrow \mathbb{R}$, for each subset $\left\{i_{1}, \ldots, i_{k+1}\right\} \subset\{1, \ldots, n\}$.

Lemma 4.2. For any exact form $\omega \in \Omega^{k+1}\left([0,1]^{n}, \partial[0,1]^{n}\right)\left(\right.$ resp., $\left.\omega \in \Omega^{k+1}\left([0,1]^{n}\right)\right)$, there is a form $\alpha \in \Omega^{k}\left([0,1]^{n}, \partial[0,1]^{n}\right)$ (resp., $\alpha \in \Omega^{k}\left([0,1]^{n}\right)$ ) given by

$$
\alpha=\sum_{\substack{I \subset[n] \\|I|=k}} \alpha_{I}(x) d x_{I},
$$

such that $d \alpha=\omega$, and for each $I,\left\|\alpha_{I}\right\|_{\text {Lip }}=\left\|d \alpha_{I}\right\|_{\infty} \leq C_{n, k}\|\omega\|_{\infty}$.

Proof. We prove this by induction on $n$ and $k$, keeping $n-k$ constant. In the base case $k=0$, we can take the function $\alpha$ to be the fiberwise integral $\int_{0}^{1} \omega$ along one of the coordinates.

To do the inductive step in the relative case, we follow the usual proof of the Poincaré lemma with compact support, following [BottTu, §1.4]. Fix a smooth bump function $\varepsilon:[0,1] \rightarrow[0,1]$ which is 0 near 0 and 1 near 1 . By applying the lemma one dimension lower, we get a form $\eta \in \Omega^{k-1}\left([0,1]^{n-1}, \partial[0,1]^{n-1}\right)$ with $d \eta=\int_{0}^{1} \omega$ and $\left\|\eta_{I}\right\|_{\text {Lip }} \leq C_{n-1, k-1}\|\omega\|_{\infty}$. Then $\omega=d \alpha$ for

$$
\alpha=\int_{0}^{t} \omega-\varepsilon\left(x_{n}\right) \pi^{*}\left(\int_{0}^{1} \omega\right)-d \varepsilon\left(x_{n}\right) \wedge \pi^{*} \eta,
$$

where $\pi$ is the projection to the $(n-1)$-cube along $x_{n}$. Notice that

$$
\alpha_{I}= \begin{cases}-\frac{d \varepsilon}{d x} \eta_{I \backslash\{n\}} & \text { if } n \in I \\ \int_{0}^{t} \omega_{I \cup\{n\}}-\varepsilon\left(x_{1}\right) \pi^{*}\left(\int_{0}^{1} \omega_{I \cup\{n\}}\right) & \text { otherwise. }\end{cases}
$$


This gives us a bound on each $\left\|\alpha_{I}\right\|_{\text {Lip }}$ in terms of the $\left\|\omega_{I}\right\|_{\text {Lip }}$ and $\left\|\eta_{I}\right\|_{\text {Lip }}$ as well as the derivatives of $\varepsilon$.

For the non-relative version, we follow the same proof, mutatis mutandis, taking

$$
\alpha=\int_{0}^{t} \omega-\pi^{*} \eta \text {. }
$$

Here and in the next section, by a random $k$-cycle we mean a random variable taking values in $k$-cycles, that is, a measure on the space of $k$-cycles. We follow the convention, common in probability theory, of blurring the distinction between a measure on a set of objects and an object randomly drawn from that measure.

Theorem 4.3. Let $Z$ be a random $k$-cycle in $\left([0,1]^{n}, \partial[0,1]^{n}\right)$ or in $[0,1]^{n}$ which satisfies the condition that for some $M>0$,

$$
\mathbb{E}(F V(Z \cap P)) \leq M
$$

for almost all $(n-k)$-planes $P$ parallel to one of the coordinate $(n-k)$-planes. Then

$$
\mathbb{E}(F V(Z)) \leq\left(\begin{array}{l}
n \\
k
\end{array}\right) C_{n, k} M
$$

where $C_{n, k}$ is the constant from Lemma 4.2.

Proof. For a $k$-form $\alpha, \int_{Z} \alpha$ depends only on $d \alpha$. Therefore to estimate (4.1) it is enough to show that for any $(k+1)$-form $\omega$ with $\|\omega\|_{\infty}=1$, there is a $k$-form $\alpha$ such that $d \alpha=\omega$ and $\int_{Z} \alpha \leq C_{n, k} M$.

By Lemma 4.2, we can choose

$$
\alpha=\sum_{\substack{I \subset[n] \\|I|=k}} \alpha_{I}(x) d x_{I}
$$

such that $\left\|d \alpha_{I}\right\|_{\infty} \leq C_{n, k}$ for every $I$. Then for $\alpha$ ranging over all these choices of antidifferentials,

$$
\begin{aligned}
F V(Z) & =\sup _{\alpha} \int_{Z} \alpha=\sup _{\alpha} \sum_{\substack{I \subset[n] \\
|I|=k}} \int_{[0,1]^{I c}} \int_{Z \cap P_{u}} \alpha_{I} d u \\
& \leq \sum_{\substack{I \subset[n] \\
|I|=k}} \int_{[0,1]^{I^{c}}}\left(\sup _{\alpha} \int_{Z \cap P_{u}} \alpha_{I}\right) d u \leq \sum_{\substack{I \subset[n] \\
|I|=k}} \int_{[0,1]^{I^{c}}} C_{n, k} F V\left(Z \cap P_{u}\right) .
\end{aligned}
$$

By linearity of expectation,

$$
\mathbb{E}(F V(Z)) \leq \sum_{\substack{I \subset[n] \\
|I|=k}} \int_{[0,1]^{I^{c}}} C_{n, k} \mathbb{E}\left(F V\left(Z \cap P_{u}\right)\right) \leq\left(\begin{array}{l}
n \\
k
\end{array}\right) C_{n, k} M .
$$

Proof of Theorem $B$, upper bound. Let $Z$ be a cycle in $C_{k}\left([0,1]^{n}, \partial[0,1]^{n}\right)$ obtained by sampling $N$ i.i.d. planes from a distribution on the set of oriented $k$-planes which intersect nontrivially with $[0,1]^{n}$, such that the distribution is uniform (with respect to Lebesgue measure on the corresponding polytope in $\mathbb{R}^{n-k}$ ) on each set of parallel planes. 
This condition clearly implies that for every coordinate $(n-k)$-plane $P, Z \cap P$ consists of at most $N$ i.i.d. positive and negative points with probability 1 . Then Theorem 3.1 implies that (4.4) holds for $Z$ with $M=C_{n-k} \operatorname{AKT}_{n-k}(N)$.

$$
\mathbb{E}(F V(Z)) \leq 2\left(\begin{array}{l}
n \\
k
\end{array}\right) C_{n, k} C_{n} \operatorname{AKT}_{n-k}(N) .
$$

Proof of Theorem $A$, upper bound. We use the fact that the transverse intersection of an oriented great $k$-sphere with an oriented great $(n-k)$-sphere is a pair of antipodal points with opposite orientations. Therefore, if $Z$ is a cycle obtained by sampling $N$ oriented great $k$-spheres independently from the uniform distribution, then for any great $(n-k)$-sphere $P$, with probability 1

$$
Z \cap P=\sum_{i=1}^{N}\left[x_{i}\right]-\left[-x_{i}\right]
$$

where the $x_{i}$ are i.i.d. uniform points on $S^{n-k}$.

Consider $S^{n}$ as a subset of $\mathbb{R}^{n+1}$, with standard unit basis vectors $e_{0}, \ldots, e_{n}$. Let $K_{i}^{ \pm}$be the preimage of the cube $[-R, R]^{n}$ under central projection (that is, projection along lines through the origin) to the plane $x_{i}= \pm 1$. If $R$ is large enough, the interiors of the $K_{i}^{ \pm}$cover $S^{n}$. Each $K_{i}^{+}$is disjoint from its antipodal set $K_{i}^{-}$; therefore for any great $(n-k)$-sphere $P$, with probability $1 Z \cap P \cap K_{i}^{ \pm}$ consists of i.i.d. uniform points. By Remark 3.2 ,

$$
\mathbb{E}\left(F V\left(Z \cap P \cap K_{i}^{ \pm}\right)\right) \leq C_{n, k} \operatorname{AKT}_{n-k}(N),
$$

where $Z \cap P \cap K_{i}^{ \pm}$is considered as a cycle in $C_{0}\left(P \cap K_{i}^{ \pm}, \partial\left(P \cap K_{i}^{ \pm}\right)\right)$.

Note that central projection sends great spheres to hyperplanes. Therefore, by Theorem 4.3, for each $i$ and sign,

$$
\mathbb{E}\left(F V\left(Z \cap K_{i}^{ \pm}\right)\right) \leq C_{n, k} \operatorname{AKT}_{n-k}(N)
$$

Set a partition of unity $\left\{\varphi_{i}^{ \pm}\right\}$subordinate to $\left\{K_{i}^{ \pm}\right\}$which is invariant with respect to the involution, that is, such that $\varphi_{i}^{+}(x)=\varphi_{i}^{-}(-x)$. To prove the theorem, it is enough, given a $k$-form $\omega \in C_{k+1}\left(S^{n}\right)$ with $\|\omega\|_{\infty}=1$, to show that for some (and therefore every) $\alpha \in C_{k}\left(S^{n}\right)$ with $d \alpha=\omega$,

$$
\int_{Z} \alpha \leq C_{n, k} \operatorname{AKT}_{n-k}(N)
$$

But note that

$$
\int_{Z} \alpha=\sum_{i=0}^{n}\left(\int_{Z \cap K_{i}^{+}} \varphi_{i}^{+} \alpha+\int_{Z \cap K_{i}^{-}} \varphi_{i}^{-} \alpha\right) .
$$

Therefore it suffices to find an antidifferential and a bound separately for each $\varphi_{i}^{ \pm} \omega$. Therefore (4.6) suffices to prove the theorem.

\section{Proof of the LOWER Bound}

Theorem 5.1. Let $Z$ be a random Lipschitz $k$-cycle in $\left([0,1]^{n}, \partial[0,1]^{n}\right)$ such that for almost every $k$-plane

$$
P_{\vec{x}}=\left\{\left(x_{1}, \ldots, x_{k}\right)\right\} \times[0,1]^{n-k} \subset[0,1]^{n},
$$


the slice $Z \cap P_{\vec{x}}$ satisfies

$$
\mathbb{E}\left(F V\left(Z \cap P_{\vec{x}}\right)\right) \geq p(\vec{x})
$$

where $p:[0,1]^{k} \rightarrow[0, \infty)$ is an $L^{1}$ function. Then

$$
\mathbb{E}(F V(Z)) \geq \int_{[0,1]^{k}} p(\vec{x}) d \vec{x} .
$$

Proof. Let $U$ be a normal current filling $Z$ such that $\operatorname{mass}(U) \leq F V(Z)+\varepsilon$, for any $\varepsilon>0$. Then for almost all $P_{\vec{x}}$, there is a slice $U \cap P_{\vec{x}}$ which fills $Z \cap P_{\vec{x}}$, and

$$
\operatorname{mass}\left(U \cap P_{\vec{x}}\right) \geq F V\left(Z \cap P_{\vec{x}}\right) .
$$

By Proposition 2.5,

$$
F V(Z)+\varepsilon \geq \operatorname{mass}(U) \geq \int_{[0,1]^{k}} \operatorname{mass}\left(U \cap P_{\vec{x}}\right) d \vec{x} \geq \int_{[0,1]^{k}} F V\left(Z \cap P_{\vec{x}}\right) d \vec{x}
$$

Since this is true for every $\varepsilon>0$, and by linearity of expectation,

$$
\mathbb{E}(F V(Z)) \geq \int_{[0,1]^{k}} \mathbb{E}\left(F V\left(Z \cap P_{\vec{x}}\right)\right) d \vec{x} \geq \int_{[0,1]^{k}} p(\vec{x}) d \vec{x} .
$$

Proof of Theorem $B$, lower bound. Let $Z$ be a cycle in $C_{k}\left([0,1]^{n}, \partial[0,1]^{n}\right)$ obtained by sampling $N$ i.i.d. planes from a distribution on the set of oriented $k$-planes which intersect nontrivially with $[0,1]^{n}$, such that the distribution is uniform (with respect to Lebesgue measure on the corresponding polytope in $\mathbb{R}^{n-k}$ ) on each set of parallel planes. Assume, perhaps by permuting coordinates, that this distribution is not concentrated on planes of the form

$$
P_{\vec{x}}=\left(x_{1}, \ldots, x_{k}\right) \times \mathbb{R}^{n-k} .
$$

As in the proof of the upper bound, it follows that for every $P_{\vec{x}}, Z \cap P_{\vec{x}}$ consists of i.i.d. positive and negative points with probability 1 . Moreover, the probability of a random plane $P$ intersecting $P_{\vec{x}}$ inside $[0,1]^{n}$ depends only on the direction of $P$ and not on $\vec{x}$. Thus

$$
\mathbb{E}\left(\operatorname{mass}\left(Z \cap P_{\vec{x}}\right)\right) \geq c N,
$$

where $c$ depends on the distribution but not on $\vec{x}$. Thus by Theorems 3.1 and 5.1 ,

$$
\mathbb{E}(F V(Z)) \geq \frac{1}{2} C_{n-k} \mathrm{AKT}_{n-k}(c N) .
$$

Proof of Theorem $A$, lower bound. Let $Z$ be a cycle in $C_{k}\left(S^{n}\right)$ obtained by sampling $N$ independent uniformly distributed great $k$-spheres. It suffices to show that for some compact submanifold $K \subset S^{n}$,

$$
F V(Z \cap K) \geq C_{n, k} \operatorname{AKT}_{n-k}(N)
$$

where $Z \cap K$ is considered as a cycle in $C_{k}(K, \partial K)$.

Recall that for any great $(n-k)$-sphere $P$, with probability 1

$$
Z \cap P=\sum_{i=1}^{N}\left[x_{i}\right]-\left[-x_{i}\right]
$$

where the $x_{i}$ are i.i.d. uniform points on $S^{n-k}$. Let $T \subset S^{n}$ be a great $k$-sphere and $T^{\prime}$ the $(n-k)$-sphere consisting of points farthest from $T$. We use $N_{\varepsilon}(U)$ to indicate the $\varepsilon$-neighborhood of the set $U$; then $S^{n} \backslash N_{\pi / 4}\left(T^{\prime}\right)=\overline{N_{\pi / 4}(T)}$ deformation retracts 
to $T$ along the orthogonal retraction $\rho: \overline{N_{\pi / 4}(T)} \rightarrow T$. We let $K=\rho^{-1}\left(K^{\prime}\right)$ where $K^{\prime}$ is some closed ball in $T$ which does not include any point and its antipode.

Notice that $K$ is foliated by equal-volume patches of great $(n-k)$-spheres $P_{u}$ which retract to points $u \in K^{\prime}$, and also does not include any point and its antipode. By Remark 3.2, there is a bilipschitz diffeomorphism from $K$ to $[0,1]^{n}$ which sends each $P_{u}$ to a plane of the form

$$
\left(x_{1}, \ldots, x_{k}\right) \times \mathbb{R}^{n-k}
$$

in a volume-preserving way (up to a constant). Therefore, for each $u \in K^{\prime}$,

$$
\mathbb{E}\left(F V\left(Z \cap P_{u} \cap K\right)\right) \geq c \operatorname{AKT}_{n-k}(c N),
$$

and applying Theorem 5.1, we obtain the result.

\section{Proof of Theorem C}

Before we prove Theorem C, we must give a more precise statement.

By an oriented $k$-pseudomanifold we mean a $k$-dimensional simplicial complex $M$ with the following properties:

- It is pure, i.e. every simplex is contained in an $k$-dimensional simplex.

- Every $k$-simplex comes with an orientation such that the sum of all the oriented $k$-simplices is a cycle in $C_{k}(M)$.

Note that this is considerably wider than the usual definition: it is just enough so that if $M$ is equipped with the standard simplexwise metric, any Lipschitz map from $M$ to a metric space $X$ defines a Lipschitz $k$-cycle in $X$.

We say $M$ has geometry bounded by $L$ if every $k$-simplex intersects at most $L$ others.

With these definitions, we restate Theorem C:

Theorem. Let $M$ be an oriented k-pseudomanifold with $N$ vertices and geometry bounded by $L$. Let $Z$ be a $k$-cycle on $[0,1]^{n}$ obtained by sending each vertex of $M$ to a uniformly random point in $[0,1]^{n}$ and extending linearly. Then there are constants $C>c>0$ depending on $n$ and $k$ such that

$$
c L^{-1} \operatorname{AKT}_{n-k}(N)<\mathbb{E}(F V(Z))<C L \operatorname{AKT}_{n-k}(N) .
$$

The concentration result will be proved in the next section.

As with Theorem B, the proof is a direct application of Theorems 4.3 and 5.1 . To apply these theorems, we need to understand the filling volumes of slices of $Z$, which is more complicated in this case because while the points are identically distributed, they are not entirely independent. We establish the upper bound in Lemma 6.3, this depends only on the fact that every point is independent of all but a constant number of others. For the lower bound in Theorem 6.13, the argument is more subtle: even if every point is correlated with only one other, such pairs could be very close and have opposite signs; then the least filling would be much smaller than for independent points. Accordingly, we have to show that most correlated points are still far apart. Together, these two bounds complete the proof.

In this section as before, fix the notation

$$
P_{\vec{x}}=\{\vec{x}\} \times[0,1]^{n-k} \subset[0,1]^{n}, \quad \vec{x} \in[0,1]^{k} .
$$

We start by analyzing the slice $Z \cap P_{\vec{x}}$. 
Lemma 6.2. Let $\vec{x} \in[0,1]^{k}$. Then the slice $Z \cap P_{\vec{x}}$ is the sum of $N$ random 0 -chains $\zeta_{1}, \ldots, \zeta_{N}$ which are identically distributed on $\left\{ \pm[y]: y \in[0,1]^{n-k}\right\} \cup\{0\}$ according to a distribution $\mu_{\vec{x}}$ depending on $k$ and $\vec{x}$. Moreover:

(i) $\mu_{\vec{x}}$ is invariant with respect to the involution sending a chain $\zeta$ to $-\zeta$;

(ii) $\mu_{\vec{x}} \leq C(n, k) \mu_{\text {Lebesgue }}$ on $[0,1]^{n-k}$.

(iii) Each $\zeta_{i}$ is independent of all but at most $L$ other $\zeta_{j}$.

Since the distribution of $Z$ is invariant under permuting coordinates, this holds for any $(n-k)$-dimensional slice in a coordinate direction.

Proof. The distribution in question is the intersection of a random linear $k$-simplex in $[0,1]^{n}$ with $P_{\vec{x}}$. Property (i) is obvious from this, and (iii) follows since a pair of $\zeta_{i}$ are independent whenever the two corresponding simplices do not intersect. To see (ii), consider the function

$$
F_{\vec{x}}:\left(\left([0,1]^{n}\right)^{k+1}, \mu_{\text {Lebesgue }}\right) \rightarrow\left(\left\{ \pm[y]: y \in[0,1]^{n-k}\right\} \cup\{0\}, \mu_{\vec{x}}\right)
$$

sending each linear $k$-simplex to its intersection with $P_{\vec{x}}$. This function is measurepreserving by definition, and its restriction to

$$
K=F_{\vec{x}}^{-1}\left\{[y]: y \in[0,1]^{n-k}\right\}
$$

is 1-Lipschitz. Therefore, by the coarea formula, the density function of $\mu_{\vec{x}}$ is given by the $[n(k+1)-(n-k)]$-dimensional Hausdorff measure of point preimages. Thus it is enough to bound $H_{(n+1) k}\left(F_{\vec{x}}^{-1}(\vec{y})\right)$ for each $\vec{y}$.

Let $T$ be the set of linear $k$-simplices with vertices in $[-1,1]^{n}$ which pass through $\overrightarrow{0}$, and let

$$
T_{\vec{x}}=(T+(\vec{x}, \overrightarrow{0})) \cap\left([0,1]^{k} \times[-1,1]^{n-k}\right)^{k+1} .
$$

(Here each vertex is translated by $(\vec{x}, \overrightarrow{0})$.) Notice that

$$
F_{\vec{x}}^{-1}(\vec{y}) \subset T_{\vec{x}}+(\overrightarrow{0}, \vec{y}) .
$$

All these translates are disjoint and their union is a subset of $\left([0,1]^{k} \times[-1,2]^{n-k}\right)^{k}$. Therefore, again by the coarea formula,

$$
H_{(n+1) k}\left(T_{\vec{x}}\right) \leq 3^{(k+1)(n-k)} .
$$

This completes the proof of (ii).

Condition (iii) gives a dependency graph of degree $\leq L$ between the $\zeta_{i}$. This graph has an $(L+1)$-coloring, giving a partition of $\{0, \ldots, N\}$ into $L+1$ disjoint subsets $I_{1}, \ldots, I_{n}$ such that for $i \in I_{j}$, the $\zeta_{i}$ are i.i.d.

6.1. Upper bound. This lemma gives the upper bound to plug into Theorem 4.3,

Lemma 6.3. For every $\vec{x} \in[0,1]^{k}$,

$$
\mathbb{E}\left(F V\left(Z \cap P_{\vec{x}}\right)\right) \leq(L+1) C_{n, k} \operatorname{AKT}_{n-k}(N) .
$$

Proof. We estimate $\mathbb{E}\left(F V\left(Z \cap P_{\vec{x}}\right)\right)$ by separately considering each summand

$$
Z(\vec{x}, j)=\sum_{i \in I_{j}} \zeta_{i}, \quad j=1, \ldots, L+1 .
$$

These summands consist of i.i.d. negative and positive points. 
Write $\nu_{\vec{x}}$ for the probability measure on $[0,1]^{n-k}$ given by

$$
\nu_{\vec{x}}(A)=\frac{\mu_{\vec{x}}\{[\vec{y}]: \vec{y} \in A\}}{\mu_{\vec{x}}\left\{[\vec{y}]: \vec{y} \in[0,1]^{n-k}\right\}} .
$$

If $\zeta$ is a random 0 -cycle in $[0,1]^{n-k}$ with $N$ positive and $N$ negative points distributed according to $\nu_{\vec{x}}$, then the AKT upper bound holds for $\zeta$ : by [BL2, equation (12)], for some constant $C_{n-k}$ independent of the measure,

$$
\mathbb{E}(F V(\zeta)) \leq C_{n-k} \operatorname{AKT}_{n-k}(N) .
$$

To reduce to this situation, we note that while $Z \cap P_{\vec{x}}$ is a cycle (and therefore has an equal number of negative and positive points), $Z(\vec{x}, j)$ may not be. We produce cycles $\tilde{Z}(\vec{x}, j)$ for $j=1, \ldots, L+1$ by adding up to $N$ additional i.i.d. points distributed according to $\nu_{\vec{x}}$. We add each point to $\tilde{Z}(\vec{x}, j)$ for two different $j$, with opposite signs, so that

$$
\sum_{j=1}^{L+1} \tilde{Z}(\vec{x}, j)=\sum_{j=1}^{L+1} Z(\vec{x}, j)=Z \cap P_{\vec{x}}
$$

Each $\tilde{Z}(\vec{x}, j)$ is a 0 -cycle consisting of at most $N$ positive and $N$ negative i.i.d. points. Therefore, by (6.4),

$$
\mathbb{E}\left(F V\left(Z \cap P_{\vec{x}}\right)\right) \leq(L+1) C_{n, k} \operatorname{AKT}_{n-k}(N) .
$$

6.2. Lower bound. For the lower bound, we begin by showing that correlated points in $Z \cap P_{\vec{x}}$ are usually not very close; this relationship is summarized in Lemma 6.5. We use this as an ingredient in the proof of Theorem 6.13, which retraces some of the steps in the original proof of the AKT theorem.

In many places, we refer to the function $F_{\vec{x}}$ and set $T_{\vec{x}}$ defined in the proof of Lemma 6.2. Also, given a subset $A \subseteq[0,1]^{n-k}$, we write

$$
[A]=\{[y]: y \in A\} \quad \text { and } \quad \pm[A]=\{ \pm[y]: y \in A\}
$$

for the respective sets of 0 -dimensional chains.

Lemma 6.5. Assume that $\vec{x} \in[1 / 4,3 / 4]^{k}$. Let $0<\ell<1 / 2$ and let $\zeta$ and $\zeta^{\prime}$ be random variables whose values are the intersections with $P_{\vec{x}}$ of two $k$-simplices $\Delta$ and $\Delta^{\prime}$ of $M$ whose vertices, some of which are shared, are chosen uniformly at random from $[0,1]^{n}$. Let $Q \subset[1 / 4,3 / 4]^{n-k}$ be a cube of side length $\ell$. Then

$$
\mathbb{P}\left[\zeta^{\prime} \in \pm[Q] \mid \zeta \in[Q]\right] \leq C_{n, k} \sqrt{\ell}
$$

Remark 6.7. A more careful analysis based on the same principle shows that

$$
\mathbb{P}\left[\zeta^{\prime} \in \pm[Q] \mid \zeta \in[Q]\right] \leq \begin{cases}C_{n, k} \ell|\log \ell| & \text { if } n-k=1, \\ C_{n, k} \ell & \text { otherwise. }\end{cases}
$$

Proof. The idea is this: suppose that $\Delta$ and $\Delta^{\prime}$ share a $(k-1)$-face $\Delta_{0}$, and let $w$ and $w^{\prime}$ be the non-shared vertices of $\Delta$ and $\Delta^{\prime}$. If the intersections of $\Delta$ and $\Delta^{\prime}$ with $P_{\vec{x}}$ are close to each other, then either the angle between $\Delta$ and $\Delta^{\prime}$ is small (forcing $w^{\prime}$ to be close to the $k$-plane containing $\Delta$ ), or $\Delta_{0}$ is close to $P_{\vec{x}}$. We would like to show that neither of these happens too often.

We will do this case in detail; the analysis when $\Delta$ and $\Delta^{\prime}$ share a lowerdimensional face is similar. 
First, we show that $\Delta_{0}$ is not very often too close to $P_{\vec{x}}$. This is easy to establish globally; the tricky bit is showing that it's true after conditioning on $\zeta$ being supported in $Q$. Let $\rho_{\vec{x}}\left(\Delta_{0}\right)$ be the distance from $\Delta_{0}$ to the hyperplane $\{\vec{x}\} \times \mathbb{R}^{n-k}$. The following lemma will be proved later.

Lemma 6.8. Let $\vec{x} \in[1 / 4,3 / 4]^{k}$ and let $A \subseteq[1 / 4,3 / 4]^{n-k}$ be a set of positive measure. Let $\Delta$ be a simplex with vertices chosen uniformly at random from $[0,1]^{n}$, and let $\Delta_{0}$ be its 0 th face. Then

$$
\mathbb{P}\left[\rho_{\vec{x}}\left(\Delta_{0}\right) \leq r \mid F_{\vec{x}}(\Delta) \in[A]\right] \leq C_{n, k} r .
$$

In particular,

$$
\mathbb{P}\left[\rho_{\vec{x}}\left(\Delta_{0}\right) \leq \sqrt{\ell} \mid \zeta \in[Q]\right] \leq C_{n, k} \sqrt{\ell} .
$$

Now we must show that when $\rho_{\vec{x}}\left(\Delta_{0}\right)>\sqrt{\ell}, \zeta^{\prime}$ doesn't very often land near $\zeta$. The point is that the difference depends on the angle between $\Delta$ and $\Delta^{\prime}$, and the distribution of this angle is not too concentrated anywhere; this is true for any given $\Delta_{0}$. Fix $\Delta_{0}$ with $\rho\left(\Delta_{0}\right) \geq \sqrt{\ell}$, and let $U\left(\Delta_{0}, Q\right) \subseteq[0,1]^{n}$ be the set of points $w^{\prime}$ such that $\zeta^{\prime} \in \pm[Q]$. Note that $U\left(\Delta_{0},\{z\}\right)$ is contained in the intersection of a $k$-plane with $[0,1]^{n(k+1)}$ and hence its $k$-dimensional Hausdorff norm is at most some $C_{n, k}$. Now we would like to use the coarea formula to integrate with respect to $z \in Q$. For this we need the following estimate, to be proved later:

Lemma 6.10. Given a linear $k$-simplex $\Delta \in\left([0,1]^{n}\right)^{k+1}$ such that at least one of its $(k-1)$-faces is at distance at least $r$ from $P_{\vec{x}}$,

$$
\sqrt{\operatorname{det}\left(\left[\left(D F_{\vec{x}}\right)_{\Delta}\right]^{T}\left(D F_{\vec{x}}\right)_{\Delta}\right)} \geq C_{n, k} r^{n-k} .
$$

Then by the coarea formula, for fixed $\Delta_{0}$,

$$
\left(\frac{\ell}{k}\right)^{\frac{n-k}{2}} \operatorname{vol}\left(U\left(\Delta_{0}, Q\right)\right) \leq C_{n, k} \operatorname{vol}(Q)
$$

and therefore

$$
\operatorname{vol}\left(U\left(\Delta_{0}, Q\right)\right) \leq C_{n, k} \ell^{\frac{n-k}{2}}
$$

Integrating this over the domain in $[0,1]^{k n}$ containing all values of $\Delta_{0}$ such that $\rho_{\vec{x}}\left(\Delta_{0}\right) \geq \sqrt{\ell}$, we see that

$$
\mathbb{P}\left[\zeta^{\prime} \in \pm[Q] \mid \zeta \in[Q], \rho\left(\Delta_{0}\right) \geq \sqrt{\ell}\right] \leq C_{n, k} \ell^{\frac{n-k}{2}} .
$$

Together, (6.9) and (6.11) imply (6.6).

Now we prove the lemmas.

Proof of Lemma 6.10. From Figure 1, we see that for every $1 \leq i \leq n-k$, there is a unit vector $\vec{v}$ such that $\left(D F_{\vec{x}}\right)_{\Delta}(\vec{v})=c \vec{e}_{i}$, for $c>r / \sqrt{k}$. Therefore

$$
\sqrt{\operatorname{det}\left(\left[\left(D F_{\vec{x}}\right)_{\Delta}\right]^{T}\left(D F_{\vec{x}}\right)_{\Delta}\right)} \geq\left(\frac{r}{\sqrt{k}}\right)^{n-k} .
$$

Proof of Lemma 6.8. Let $X$ be the event that $\rho_{\vec{x}}(\Delta) \leq r$, and let $Y$ be the event that $\Delta \cap P_{\vec{x}} \in[Q]$. Bayes' rule states that

$$
\mathbb{P}(X \mid Y)=\mathbb{P}(Y \mid X) \mathbb{P}(X) / \mathbb{P}(Y) .
$$




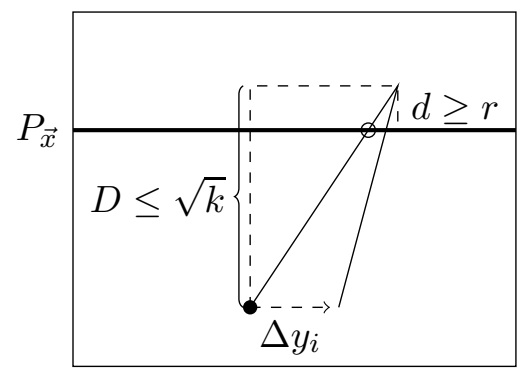

Figure 1 . When a vertex moves by $\Delta y_{i}, \Delta \cap P_{\vec{x}}$ moves by $(d / D) \Delta y_{i}$.

We will bound $\mathbb{P}(X \mid Y)$ by giving upper bounds for $\mathbb{P}(Y \mid X)$ and $\mathbb{P}(X)$ and a lower bound for $\mathbb{P}(Y)$.

We first show that

$$
\mathbb{P}(Y \mid X) \leq 3^{(n-k)(k+1)} \operatorname{vol}(A)
$$

In fact, in this inequality, $X$ may be any constraint on the first $k$ coordinates of each vertex of $\Delta$ (note that $\rho_{\vec{x}}(\Delta)$ depends only on those coordinates).

We start by fixing the first $k$ coordinates of each vertex. Given a simplex $\Delta$ with vertices in $\mathbb{R}^{n}$, let $\pi(\Delta)$ be the projection onto the first $k$ coordinates, and fix a simplex $\Delta_{\pi}$ with vertices in $[0,1]^{k}$. Let $U \subset\left([-1,1]^{n-k}\right)^{k+1}$ contain the last $n-k$ coordinates of simplices in $\pi^{-1}\left(\Delta_{\pi}\right)$ whose intersection with $P_{\vec{x}}$ is $[(\vec{x}, \overrightarrow{0})]$. Write $U+A$ to mean the set of translates of simplices in $U$ by points in $A$. Notice that

(1) $U+[0,1]^{n-k} \subseteq\left([-1,2]^{n-k}\right)^{k+1}$,

(2) the volume of $U+A$ is proportional to $\operatorname{vol}(A)$, and

(3) $U+\{\vec{y}\}$ contains $F^{-1}([\vec{y}]) \cap \pi^{-1}\left(\Delta_{\pi}\right)$.

Therefore, the probability that a given point of $\left([0,1]^{n-k}\right)^{k+1}$ is contained in $U+A$ is at most $3^{(n-k)(k+1)} \operatorname{vol}(A)$, and this in turn bounds

$$
\operatorname{vol}\left(F^{-1}([A]) \cap \pi^{-1}\left(\Delta_{\pi}\right)\right) .
$$

Integrating over possible values of $\Delta_{\pi}$ gives (6.12).

Now we show that $\mathbb{P}(X) \leq C_{n, k} r$. Choosing $k$ points in $\mathbb{R}^{k}$ uniformly at random induces a probability measure on the set of $(k-1)$-planes, whose density at a plane $P$ is proportional to $\operatorname{vol}\left(P \cap[0,1]^{k}\right)^{k}$. This density is bounded above by some $C_{n, k}$, and so the set of planes whose distance from $\vec{x}$ is at most $r$ has measure $\leq C_{n, k} r$.

Finally we must give a lower bound for $\mathbb{P}(Y)$, that is, the volume of

$$
\Sigma_{Y}=\left\{\Delta: F_{\vec{x}}(\Delta) \in[A]\right\} \subseteq[0,1]^{n(k+1)} .
$$

For this, we note that when $\vec{x}$ and $\vec{y}$ have all coordinates in $[1 / 4,3 / 4]$,

$$
F_{\vec{x}}^{-1}([\vec{y}]) \supseteq T_{\vec{x}} \cap\left([-1 / 4,1 / 4]^{n}+\{(\vec{x}, \overrightarrow{0})\}\right)+\{(\overrightarrow{0}, \vec{y})\} .
$$

This is a translate of a set which is independent of $\vec{x}$ and $\vec{y}$. Taking all the translates with respect to vectors in $A$, we see that $\Sigma_{Y}$ contains a set whose volume depends linearly on $\operatorname{vol}(A)$ and is easily seen to be positive.

Thus overall we get $\mathbb{P}(X \mid Y) \leq C_{n, k} r$.

Finally we have the tools we need to prove the lower bound for Theorem $\mathrm{C}$. 
Theorem 6.13. For every $\vec{x} \in[1 / 4,3 / 4]^{k}$,

$$
\mathbb{E}\left(F V\left(Z \cap P_{\vec{x}}\right)\right) \geq c_{n, k} L^{-1} \operatorname{AKT}_{n-k}(N) .
$$

Proof. Write $d=n-k$. We are trying to find a lower bound for the expected filling length of a certain distribution on 0 -cycles in $[0,1]^{d}$ which is defined as a sum of a large number of identically distributed points. This is very similar to the original AKT theorem, in which the points are, in addition, independent and uniformly distributed. In [AKT], the lower bound is proved using the dual definition of filling volume given in (4.1), which we restate here for a 0 -cycle $Z_{0}$ in $[0,1]^{d}$ :

$$
F V\left(Z_{0}\right)=\sup \left\{\int_{Z_{0}} f:[0,1]^{d} \rightarrow \mathbb{R} \text { such that } \operatorname{Lip}(f) \leq 1\right\} .
$$

For the case $d=2$, Ajtai, Komlós and Tusnády construct a $\sqrt{N \log N \text {-Lipschitz }}$ function $f$ whose integral over $Z_{0}$ is usually at least $c N \log N$. The filling volume $F V\left(Z_{0}\right)$ is then bounded below by the ratio of the integral to the Lipschitz constant.

When $d=2$, we construct $f$ the same way as in [AKT], but on a modified point set in order to overcome two issues:

(1) The points of $Z \cap P_{\vec{x}}$ are not uniformly distributed. We use a bilipschitz rescaling of the domain to make sure that they are.

(2) The points of $Z \cap P_{\vec{x}}$ are not independent. We instead construct the function $f$ to have a large integral over a large independent subset, and then use Lemma 6.5 to show that the integral over the remaining points is small.

For $d=1$ and $d \geq 3$, we use the same reduction steps, but the function $f$ is somewhat simpler. We start by more precisely describing the common argument and then give the detailed construction of $f$ for each case.

Let $\zeta_{i}$ be the chain-valued random variables corresponding to intersections of $k$ simplices of $Z$ with $P_{\vec{x}}$. Recall that $\zeta_{i}= \pm\left[v_{i}\right]$ or $\{0\}$, with $v_{i} \in[0,1]^{d}$ identically distributed according to a density which is bounded below on $[1 / 4,3 / 4]^{d}$. Moreover, this bound is uniform with respect to $\vec{x} \in[1 / 4,3 / 4]^{k}$. Thus, following Remark [3.2, there is a uniformly bilipschitz family of diffeomorphisms

$$
\varphi_{\vec{x}}:[1 / 4,3 / 4]^{d} \rightarrow[0,1]^{d}
$$

which send this density to a constant times the standard volume form. In particular, Lemma 6.5 still holds for the $\zeta_{i}$ after applying the diffeomorphism. We now write $\zeta_{i}$ for $\varphi_{\vec{x}}\left(\zeta_{i}\right)$.

In each case, consider an $(L+1)$-coloring $I_{1}, \ldots, I_{L+1}$ of the dependency graph between the $\zeta_{i}$, with the colored subsets ordered from largest to smallest. Note that each of the $\zeta_{i}$ is correlated with $\zeta_{i^{\prime}}$ for at most $L$ values of $i^{\prime}$.

We write $Z_{j}=\sum_{i \in I_{j}} \zeta_{i}$. In each case, we show that $Z_{1}$ is hard to fill by constructing a Lipschitz function $f$ such that

$$
\int_{Z_{1}} f \geq \frac{c_{n, k}}{L} \operatorname{AKT}_{n-k}(N) \operatorname{Lip} f \text { with high probability. }
$$

Then we show that $\mathbb{E}\left(\int_{Z_{j}} f\right)$ for each $j \neq 1$ is small enough that it does not affect the overall asymptotics.

In each case, the function $f$ is constructed as a sum of simpler functions. Given a cube $Q \subseteq[0,1]^{d}$, let $\Delta_{Q}:[0,1]^{d} \rightarrow \mathbb{R}$ be the function supported on $Q$ whose graph is a symmetric pyramid with base $Q$ and height 1 . We also write $\Delta_{v}^{r}$ for the cube in 
the lattice of side length $r$ which contains $v \in[0,1]^{d}$. The function $f$ will consist of a sum of scaled copies of $\Delta_{Q}$, each reflecting the "imbalance" of positive and negative points on the cube $Q$. The main difference between different dimensions is the scale of these cubes: for $d \geq 3$, the cubes are at the smallest scale (comparable to the average distance between neighboring points), for $d=1$ they are at the largest scale (comparable to 1 ), and for $d=2$ we use cubes at many scales, as in the original proof of AKT.

In each case, we construct $f$ by means of an auxiliary function

$$
g(x)=\sum_{i \in I_{1}} g_{\zeta_{i}}(x)
$$

(in the case $d=2$, each $\zeta_{i}$ is associated to many summands $g_{\zeta_{i}}^{r}$ at different scales, which we consider separately). This $g$ may not satisfy the desired upper bound on the Lipschitz constant, so we remove some of the summands where they are too concentrated to produce $f$. Whenever $\zeta_{i}$ is independent from $\zeta_{i^{\prime}}, \mathbb{E}\left(\int_{\zeta_{i^{\prime}}} g_{\zeta_{i}}(x)\right)=0$, and so for every $j \neq 1$ we can write

$$
\left|\mathbb{E}\left(\int_{Z_{j}} g\right)\right| \leq \sum_{i \in I_{j}} \sum\left\{\left|\mathbb{E}\left(\int_{\zeta_{i}} g_{\zeta_{i^{\prime}}}\right)\right| \mid \zeta_{i^{\prime}} \text { is correlated with } \zeta_{i}\right\} .
$$

By Lemma 6.5, this correlation is not too high, and therefore we can bound each of the summands. By the construction of $f$, this also bounds $\left|\mathbb{E}\left(\int_{Z_{j}} f\right)\right|$.

If we tune everything correctly, we get that for each $j \neq 1$,

$$
\left|\mathbb{E}\left(\int_{Z_{j}} f\right)\right| \leq \frac{1}{2 L} \mathbb{E}\left(\int_{Z_{1}} f\right)
$$

giving a lower bound on $\mathbb{E}\left(\int_{Z} f\right)$.

Case $d \geq 3$. We split the cube $[0,1]^{d}$ into $\sim N$ subcubes of side length $r \approx N^{-1 / d}$, and let

$$
\begin{aligned}
& g(x)=\sum_{i \in I_{1}} g_{i}(x)=\sum_{i \in I_{1}} \pm r \Delta_{v_{i}}^{r}(x) \text { where } \zeta_{i}= \pm\left[v_{i}\right], \\
& f(x)=\sum_{t_{1}, \ldots, t_{d}=0}^{r^{-1}-1} \operatorname{sign}\left(\int_{Z_{1}} \chi_{Q_{r}\left(t_{1}, \ldots, t_{d}\right)}\right) r \Delta_{Q_{r}\left(t_{1}, \ldots, t_{d}\right)}(x),
\end{aligned}
$$

where $Q_{r}\left(t_{1}, \ldots, t_{d}\right)$ is the cube with side length $r$ whose vertex closest to the origin is $\left(r t_{1}, \ldots, r t_{d}\right)$. Note that $f$ is 2 -Lipschitz.

We can think of the number of points landing in each subcube as $N$ independent $\lambda=1$ Poisson processes which we stop once their sum reaches roughly

$$
\mathbb{P}\left(\zeta_{i} \cap[0,1]^{d} \neq 0\right)\left|I_{1}\right| \text {. }
$$

By the law of large numbers, the stopping time will be very close to

$$
t=N^{-1} \mathbb{P}\left(\zeta_{i}= \pm[y], y \in[0,1]^{d}\right)\left|I_{1}\right|
$$

and very nearly $N t e^{-t}$ of the subcubes will contain exactly one point. Of these, with high probability, at least $(1 / 2) \cdot 3^{-d} \cdot N t e^{-t}$ will be contained in the middle third of their subcube. Therefore,

$$
\int_{Z_{1}} f \geq \frac{c_{n, k}}{L} \cdot N^{\frac{d-1}{d}} \text { with high probability. }
$$


On the other hand, given $j \neq 1, i \in I_{j}$, and $i^{\prime} \in I_{1}$ such that $\zeta_{i}$ is correlated with $\zeta_{i^{\prime}}$, Lemma 6.5 tells us that

$$
\left|\mathbb{E}\left(\int_{\zeta_{i}} g_{i^{\prime}}\right)\right| \leq C_{n, k} r^{3 / 2}
$$

and therefore

$$
\left|\mathbb{E}\left(\int_{Z_{j}} f\right)\right| \leq \sum_{i \in I_{j}} L C_{n, k} r^{3 / 2} \leq L C_{n, k} N^{\frac{d-1}{d}-\frac{1}{2 d}} .
$$

Since this is small compared to $\int_{Z_{1}} f$, this shows that

$$
\mathbb{E}\left(\int_{Z \cap P_{\vec{x}}} f\right) \geq \frac{c_{n, k}}{L} N^{\frac{d-1}{d}} \operatorname{Lip} f \quad \text { for large enough } N .
$$

Case $d=2$. This case is broadly similar, but we build the function $f$ in a more complicated way, following the original proof of [AKT]. For an integer $r$, let $Q_{s t}^{r}$ be the square of side length $2^{-r}$ whose lower left corner is at $\left(s \cdot 2^{-r}, t \cdot 2^{-r}\right)$, and write $\Delta_{s t}^{r}=\Delta_{Q_{s t}^{r}}$. We write

$$
\begin{aligned}
g(x, y) & =\sum_{r=1}^{0.1 \log N} \sum_{s, t=0}^{2^{r}-1} g_{s t}^{r}(x, y)=\sum_{r=1}^{0.1 \log N} \sum_{s, t=0}^{N} \Delta_{s t}^{r}(x, y) \int_{Z_{1}} \Delta_{s t}^{r} \\
& =\sum_{r=1}^{0.1 \log N} \sum_{i \in I_{1}} g_{\zeta_{i}}^{r}(x, y) \\
& =\sum_{r=1}^{0.1 \log N} \sum_{i \in I_{1}} \Delta_{v_{i}}^{r}(x, y) \int_{\zeta_{i}} \Delta_{v_{i}}^{r} \quad \text { where } \zeta_{i}= \pm\left[v_{i}\right] .
\end{aligned}
$$

Notice that $\int_{Z_{1}} g_{s t}^{r}$ is always nonnegative: roughly speaking, it measures the square of the "imbalance" of positive and negative points in $Q_{s t}^{r}$. In particular, it's not hard to see that $\mathbb{E}\left(\int_{Z_{1}} g_{s t}^{r}\right)=c_{n, k} \cdot 2^{-2 r} N$, and therefore $\mathbb{E}\left(\int_{Z_{1}} g\right)=c_{n, k} N \log N$.

On the other hand, the derivative of $g$ is $O(\sqrt{N \log N})$ on average, but can be much larger in some places. To remedy this, Ajtai, Komlós, and Tusnády introduced a "stopping time" rule, building $f$ as the sum of some, but not all of the $g_{s t}^{r}$. We do not need to give the exact definition, remarking only that the function $f$ satisfies

$$
\begin{aligned}
\mathbb{E}\left(\int_{Z_{1}} f\right) & \geq \frac{c_{n, k}}{L} N \log N \\
\operatorname{Lip} f & \leq C_{n, k} \sqrt{N \log N} .
\end{aligned}
$$

Now, for $j \neq 1$,

$$
\left|\mathbb{E}\left(\int_{Z_{j}} f\right)\right| \leq \sum_{i \in I_{j}} \sum_{r=1}^{0.1 \log N} \sum\left\{\left|\mathbb{E}\left(\int_{\zeta_{i}} g_{\zeta_{i^{\prime}}}^{r}\right)\right| \mid \zeta_{i^{\prime}} \text { is correlated with } \zeta_{i}\right\} .
$$

By Lemma 6.5, the value of each term of this triple sum is $O\left(2^{-r / 2}\right)$. Therefore,

$$
\left|\mathbb{E}\left(\int_{Z_{j}} f\right)\right| \leq C_{n, k} L N \sum_{r=1}^{0.1 \log N} 2^{-r / 2} \leq(1+\sqrt{2}) C_{n, k} L N .
$$

Combining this with (6.14) and (6.15), we see that

$$
\mathbb{E}\left(\int_{Z \cap P_{\vec{x}}} f\right) \geq \frac{c_{n, k}}{L} \sqrt{N \log N} \operatorname{Lip} f \quad \text { for large enough } N .
$$


Case $d=1$. We split the interval $[0,1]$ into $R$ equal regions, with $R$ to be determined later. Write $\Delta_{s}=\Delta_{[s / R,(s+1) / R]}$, and let

$$
g(x)=\sum_{s=0}^{R-1} \Delta_{s}(x) \int_{Z_{1}} \chi_{\left[\frac{s}{R}, \frac{s+1}{R}\right]}=\sum_{i \in I_{1}} g_{\zeta_{i}}(x)=\sum_{i \in I_{1}} \pm \Delta_{y_{i}}^{1 / R}(x) \quad \text { where } \zeta_{i}= \pm\left[y_{i}\right] .
$$

We obtain the desired function $f$ by replacing $\int_{Z_{1}} \chi_{\left[\frac{s}{R}, \frac{s+1}{R}\right]}$ with

$$
h_{s}=\operatorname{sign}\left(\int_{Z_{1}} \chi_{\left[\frac{s}{R}, \frac{s+1}{R}\right]}\right) \min \left\{\left|\int_{Z_{1}} \chi_{\left[\frac{s}{R}, \frac{s+1}{R}\right]}\right|, C_{n, k} \sqrt{\frac{N}{R}}\right\}
$$

for some sufficiently large $C_{n, k}$. Then $\operatorname{Lip} f \leq C_{n, k} \sqrt{N R}$ and $\int_{Z_{1}} f \geq \frac{c_{n, k}}{L} N$.

On the other hand, for $j \neq 1$ and any $i \in I_{j}$ and $i^{\prime} \in I_{1}$ such that $\zeta_{i}$ and $\zeta_{i^{\prime}}$ are correlated, by Lemma 6.5, $\left|\mathbb{E}\left(\int_{\zeta_{i}} g_{\zeta^{\prime}}\right)\right| \leq C_{n, k} R^{-1 / 2}$, and therefore

$$
\left|\mathbb{E}\left(\int_{Z_{j}} f\right)\right| \leq C_{n, k} L N R^{-1 / 2}
$$

For some large enough $R$, depending on $n$ and $k$ but not on $N$,

$$
\left|\mathbb{E}\left(\int_{Z_{j}} f\right)\right| \leq \frac{1}{2 L^{2}} \mathbb{E}\left(\int_{Z_{1}} f\right) .
$$

Thus $\mathbb{E}\left(\int_{Z \cap P_{\vec{x}}} f\right) \geq C_{n, k} \sqrt{N} \operatorname{Lip} f$, completing the proof.

\section{Concentration of measure}

In this section, we show that when $n-k \geq 2$, the size of the filling tends to concentrate around its mean. That is, we show that (1.2) holds in the case of Theorems A, B, and C]. We first prove this in the case of Theorem 3.1, The main tool is the concentration of measure in high-dimensional balls, an idea due to Gromov and Milman [GM] and of wide importance in probability theory [Led]. We follow the exposition due to Bobkov and Ledoux [BL1, §7.1] which covers the 1-dimensional case; the higher-dimensional cases are essentially the same although they do not seem to appear explicitly in the literature.

Theorem 7.1. Let $Z$ be a random cycle in $C_{0}\left([0,1]^{n}, \partial[0,1]^{n}\right)$ as in Theorem 3.1 . Then for every $r>0$,

$$
\mathbb{P}[|F V(Z)-\mathbb{E}(F V(Z))| \geq r] \leq C_{1} \exp \left(-C_{2} r / \sqrt{N}\right)
$$

for universal constants $C_{1}, C_{2}>0$.

In particular, the standard deviation of $F V(Z)$ is at most $O(\sqrt{N})$. In other words, for $n \geq 2, F V(Z) / \mathbb{E}(F V(Z))$ converges to 1 as $N \rightarrow \infty$.

Proof. Equip $X=C_{0}\left([0,1]^{n}, \partial[0,1]^{n}\right)$ with the metric

$$
d_{F V}\left(Z, Z^{\prime}\right)=F V\left(Z-Z^{\prime}\right)
$$

and let $E=[-1,1] \times[0,1]^{n-1}$. Define $\zeta_{0}: E \rightarrow X$ by

$$
\zeta_{0}\left( \pm x_{1}, x_{2}, \ldots, x_{n}\right)= \pm\left[\left(x_{1}, x_{2}, \ldots, x_{n}\right)\right]
$$


and $\zeta:\left(E^{N}, d_{\text {Eucl }}\right) \rightarrow X$ by

$$
\zeta\left(v_{1}, \ldots, v_{N}\right)=\sum_{i=1}^{N} \zeta_{0}\left(v_{i}\right) .
$$

This map is $\sqrt{N}$-Lipschitz since when every point moves by a tiny amount $\varepsilon$, the distance is $\sqrt{N} \varepsilon$ in the domain and $N \varepsilon$ in the range.

Define the concentration function of a metric measure space $(M, d, \mu)$ of total measure 1 to be

$$
\operatorname{conc}_{M}(r)=\sup \left\{1-\mu\left(N_{r}(A)\right) \mid \mu(A) \geq 1 / 2\right\}, \quad r>0,
$$

where $N_{r}(A)$ is the $r$-neighborhood of the set $A$. The key observation of Gromov and Milman [GM, Thm. 4.1] is that

$$
\operatorname{conc}_{M}(r) \leq \frac{3}{4} e^{-\ln (3 / 2) \lambda_{1} r},
$$

where $\lambda_{1}$ is the first nonzero eigenvalue of the Laplacian on $M$. Since the spectrum of a product of manifolds is the sum of its spectra, $\lambda_{1}$ is constant on powers of $M$. The map $\zeta$ is measure-preserving, so it follows that

$$
\operatorname{conc}_{X}(r) \leq \frac{3}{4} \exp \left(-\ln (3 / 2) \lambda_{1} r / \sqrt{N}\right) \text {. }
$$

Therefore, for any 1-Lipschitz function $u: X \rightarrow \mathbb{R}$,

$$
\mathbb{P}[|u(Z)-\operatorname{median}(u)| \geq r] \leq \frac{3}{2} \exp \left(-\ln (3 / 2) \lambda_{1} r / \sqrt{N}\right) .
$$

By Chebyshev's inequality, the same, modulo constants, holds for the mean (see also [Led, Prop. 1.10]).

To adapt this proof for the case of Theorems $\mathrm{A}, \mathrm{B}$, and C, we just have to change the space $E$ : take

$$
\begin{aligned}
E_{\text {sphere }} & =\widetilde{G r}_{k}\left(\mathbb{R}^{n}\right) & & \text { for Theorem } \mathrm{A} \\
E_{\text {cube }} & =\left\{\text { affine } k \text {-planes } P \subset \mathbb{R}^{n} \mid P \cap[0,1]^{n} \neq \emptyset\right\} & & \text { for Theorem B } \\
E_{\text {knot }} & =[0,1]^{n} & & \text { for Theorem C. }
\end{aligned}
$$

In the first two cases, the map $\zeta$ is constructed as before. In the last case, for a $k$ pseudomanifold $M$ with vertex set $M^{0}, \zeta_{M}: E_{\mathrm{knot}}^{\left|M^{0}\right|} \rightarrow Z_{k}\left([0,1]^{n}\right)$ sends $\left(v_{1}, \ldots, v_{\left|M^{0}\right|}\right)$ to the image of the linear immersion of $M$ with vertices $\left(v_{1}, \ldots, v_{\left|M^{0}\right|}\right)$.

In each case, it is easy to see that if the space of $k$-cycles is given the filling volume metric, then $\zeta$ is $\sqrt{N}$-Lipschitz. Therefore, the rest of the proof is identical to that of Theorem 7.1 .

\section{REFERENCES}

$\left[\mathrm{ABD}^{+}\right]$J. Arsuaga, T. Blackstone, Y. Diao, E. Karadayi, and M. Saito, Linking of uniform random polygons in confined spaces, J. Phys. A 40 (2007), no. 9, 1925-1936.

[AKT] M. Ajtai, J. Komlós, and G. Tusnády, On optimal matchings, Combinatorica 4 (1984), no. 4, 259-264.

[Bany] A. Banyaga, Formes-volume sur les variétés à bord, Enseign. Math. (2) 20 (1974), 127131.

[BL1] S. Bobkov and M. Ledoux, One-dimensional empirical measures, order statistics, and Kantorovich transport distances, Mem. Amer. Math. Soc. 261 (2019), no. 1259, v+126. 
[BL2] A simple Fourier analytic proof of the AKT optimal matching theorem, arXiv:1909.06193 [math.PR], 2019.

[BMPR] M. Bruveris, P. W. Michor, A. Parusiński, and A. Rainer, Moser's theorem on manifolds with corners, Proc. Amer. Math. Soc. 146 (2018), no. 11, 4889-4897.

[BottTu] R. Bott and L. W. Tu, Differential forms in algebraic topology, Graduate Texts in Mathematics, no. 82, Springer, 1982.

$\left[\mathrm{EPC}^{+}\right]$D. Epstein, M. Paterson, J. Cannon, D. Holt, S. Levy, and W. P. Thurston, Word processing in groups, Jones and Bartlett, 1992.

[E-Z] Ch. Even-Zohar, Models of random knots, J. Appl. Comput. Topol. 1 (2017), no. 2, 263296.

[Fed] H. Federer, Geometric measure theory, Die Grundlehren der mathematischen Wissenschaften, Band 153, Springer-Verlag New York Inc., New York, 1969.

[FF] H. Federer and W. H. Fleming, Normal and integral currents, Ann. of Math. (2) 72 (1960), 458-520.

[FK] E. Flapan and K. Kozai, Linking number and writhe in random linear embeddings of graphs, J. Math. Chem. 54 (2016), no. 5, 1117-1133.

[FKW] P. Franek, M. Krčál, and H. Wagner, Solving equations and optimization problems with uncertainty, J. Appl. Comput. Topol. 1 (2018), no. 3-4, 297-330.

[GM] M. Gromov and V. D. Milman, A topological application of the isoperimetric inequality, American Journal of Mathematics 105 (1983), no. 4, 843-854.

[Gro] M. Gromov, Homotopical effects of dilatation, J. Differential Geom. 13 (1978), no. 3, 303-310.

[HPZ] N. Holden, Y. Peres, and A. Zhai, Gravitational allocation on the sphere, Proc. Natl. Acad. Sci. USA 115 (2018), no. 39, 9666-9671.

[HS] R. Hardt and L. Simon, Boundary regularity and embedded solutions for the oriented Plateau problem, Ann. of Math. (2) 110 (1979), no. 3, 439-486.

[Jones] P. W. Jones, Rectifiable sets and the traveling salesman problem, Invent. Math. 102 (1990), no. $1,1-15$.

[Led] M. Ledoux, The concentration of measure phenomenon, Mathematical Surveys and Monographs, vol. 89, American Mathematical Society, Providence, RI, 2001.

[Marko] J. F. Marko, Linking topology of tethered polymer rings with applications to chromosome segregation and estimation of the knotting length, Phys. Rev. E 79 (2009), 051905.

[Mil] K. C. Millett, Monte Carlo explorations of polygonal knot spaces, Knots in Hellas '98 (Delphi), Ser. Knots Everything, vol. 24, World Sci. Publ., River Edge, NJ, 2000, pp. 306334 .

[Mor] F. Morgan, Geometric measure theory: A beginner's guide, fourth ed., Elsevier/Academic Press, Amsterdam, 2009.

[Moser] J. Moser, On the volume elements on a manifold, Trans. Amer. Math. Soc. 120 (1965), 286-294.

[Shor] P. W. Shor, The average-case analysis of some on-line algorithms for bin packing, Combinatorica 6 (1986), no. 2, 179-200.

[Tal1] M. Talagrand, Matching random samples in many dimensions, Ann. Appl. Probab. 2 (1992), no. 4, 846-856.

[Tal2] The transportation cost from the uniform measure to the empirical measure in dimension $\geq 3$, Ann. Probab. 22 (1994), no. 2, 919-959.

[Tal3] U Upper and lower bounds for stochastic processes: Modern methods and classical problems, Ergebnisse der Mathematik und ihrer Grenzgebiete. 3. Folge. A Series of Modern Surveys in Mathematics, vol. 60, Springer, Heidelberg, 2014.

[Tanaka] F. Tanaka, Gauge Theory of Topological Entanglements. I: - General Theory -, Progress of Theoretical Physics 68 (1982), no. 1, 148-163.

[Young] R. Young, Quantitative nonorientability of embedded cycles, Duke Math. J. 167 (2018), no. $1,41-108$.

Department of Mathematics, UCSB, Santa Barbara, California, USA

Email address: manin@math.ucsb.edu 